OPEN ACCESS

Edited by:

Sha Wu,

Southern Medical University, China

Reviewed by:

Yuan Cheng,

Peking University People's Hospital,

China

DiXie,

Yale University, United States

*Correspondence:

Tailang Yin

reproductive@whu.edu.cn

Yan Zhang

peneyyan@mail.ustc.edu.cn

Lianghui Diao

I.diao@pku.edu.cn

${ }^{\text {t}}$ These authors have contributed equally to this work

Specialty section:

This article was submitted to

Immunological Tolerance and Regulation,

a section of the journal

Frontiers in Immunology

Received: 30 May 2021

Accepted: 24 August 2021

Published: 09 September 2021

Citation:

Wei Y, Ding J, Li J, Cai S, LiU S, Hong $L$, Yin $T$, Zhang $Y$ and Diao $L$ (2021) Metabolic Reprogramming of

Immune Cells at the Maternal-Fetal

Interface and the Development of

Techniques for Immunometabolism.

Front. Immunol. 12:717014.

doi: 10.3389/fimmu.2021.717014

\section{Metabolic Reprogramming of Immune Cells at the Maternal-Fetal Interface and the Development of Techniques for Immunometabolism}

\author{
Yiqiu Wei ${ }^{1 \dagger}$, Jinli Ding ${ }^{1 \dagger}$, Jianan $\mathrm{Li}^{1}$, Songchen $\mathrm{Cai}^{2}$, Su Liu ${ }^{2,3}$, Ling Hong ${ }^{2,3}$, \\ Tailang Yin ${ }^{1 *}$, Yan Zhang ${ }^{* *}$ and Lianghui Diao ${ }^{2,3 *}$ \\ ${ }^{1}$ Reproductive Medicine Center, Renmin Hospital of Wuhan University, Wuhan, China, ${ }^{2}$ Shenzhen Key Laboratory for \\ Reproductive Immunology of Peri-Implantation, Shenzhen Zhongshan Institute for Reproduction and Genetics, Shenzhen \\ Zhongshan Urology Hospital, Shenzhen, China, ${ }^{3}$ Shenzhen Jinxin Medical Technology Innovation Center, Co., Ltd., \\ Shenzhen, China, ${ }^{4}$ Department of Clinical Laboratory, Renmin Hospital of Wuhan University, Wuhan, China
}

Immunity and metabolism are interdependent and coordinated, which are the core mechanisms for the body to maintain homeostasis. In tumor immunology research, immunometabolism has been a research hotspot and has achieved groundbreaking changes in recent years. However, in the field of maternal-fetal medicine, research on immunometabolism is still lagging. Reports directly investigating the roles of immunometabolism in the endometrial microenvironment and regulation of maternalfetal immune tolerance are relatively few. This review highlights the leading techniques used to study immunometabolism and their development, the immune cells at the maternal-fetal interface and their metabolic features required for the implementation of their functions, explores the interaction between immunometabolism and pregnancy regulation based on little evidence and clues, and attempts to propose some new research directions and perspectives.

Keywords: maternal-fetal tolerance, metabolomics, cell metabolism analysis, reproductive immunology, natural killer cells, macrophages, $T$ cells

\section{INTRODUCTION}

Classical reproductive immunology considers that pregnancy can be understood as a model for "allograft transplantation." The establishment of a normal pregnancy depends on maternal immune recognition and tolerance to the embryonic alloantigens (1). The interaction between immune cells and cytokines at the maternal-fetal interface creates an immune tolerance microenvironment, preventing the fetus from the maternal immune system attacks and maintaining a certain degree of immunity to protect the mother from harmful pathogens $(2,3)$. The immune microenvironment at the maternal-fetal interface is mainly composed of decidual stromal cells (DSC), decidual epithelial cells (DEC), trophoblast cells, decidua immune cells (DIC), and the soluble factors. The establishment of pregnancy induces a series of maternal immune-inflammatory responses and adaptive changes in material and energy metabolism (4). With the progress of pregnancy, the maternal-fetal interface immune microenvironment shows a dynamic equilibrium: during early 
pregnancy, the maternal-fetal interface changes from an inflammatory microenvironment that is conducive to embryo implantation and pregnancy establishment to an immune tolerance microenvironment that is conducive to pregnancy maintenance and fetal growth and development. Until late pregnancy, it gradually tends to inflammatory transition, and is endogenously prepared for delivery. When the regulation of maternal-fetal immune tolerance is disturbed, the result can be an excessive immune response to the fetus and the development of immune rejection, leading to pathological pregnancy or even pregnancy loss.

Immune cell metabolic reprogramming is a general term for the response of immune cells to crucial changes in the environment, involving changes in critical metabolic enzymes, metabolites, and metabolic pathways. Various cytokines and antigens can regulate the metabolism of immune cells. Then, metabolic reprogramming occurs and affects the outcome of the immune response. The metabolic level of the cell itself is also closely related to its function (5-7). Many immune cells are infiltrated in the uterus, and the success of pregnancy immunity depends to a certain extent on their crosstalk with trophoblast cells (8). Immune cells, such as natural killer $(\mathrm{NK})$ cells, macrophages $(\mathrm{M} \varphi)$, T cells and dendritic cells (DC), exhibit specific phenotypes and functions through a series of cell differentiation and participate in the regulation of reproductive processes (9). In addition to meeting the material basis required for the growth and development of the fetus, metabolic reprogramming also plays a role in regulating the immune state. Finally, it establishes an "immune tolerance" microenvironment in the uterus, achieving "peaceful coexistence" between mother and fetus (10). However, the differentiation and regulatory mechanism of endometrial immune cells remain to be elucidated. Whether immunometabolism regulates the phenotype and function of immune cells and regulates the physiological processes of reproduction remains further revealed. This review focuses on the leading techniques used to study immunometabolism and their development, summarizes the functions of immune cells at the maternal-fetal interface, and explores the potential role of immune cell metabolic reprogramming in the maternal-fetal interface to provide new ideas for the diagnosis and treatment of clinical pregnancy-related diseases.

\section{LEADING TECHNIQUES FOR DETECTION OF IMMUNE CELL METABOLISM}

Metabolism is a crucial physiological activity in cells. There are six main metabolic pathways in immune cells, including glycolysis, tricarboxylic acid (TCA) cycle, pentose phosphate pathway (PPP), fatty acid oxidation (FAO), fatty acid synthesis (FAS), and amino acid metabolism (10). Their interaction provides energy and nutrients for the maintenance of cell life activities (Figure 1). Cell metabolism is more than just a process of adenosine triphosphate (ATP) production, biosynthesis, and catabolism. Metabolites and metabolic fluxes can regulate cellular signaling pathways and post-translational modifications (PTM) (11), leading to changes in gene expression and even in the epigenome. For example, post-translational histone modification can be affected by changing local concentrations of key metabolites, thereby regulating transcription and other DNAtemplated functions (12). Metabolic activity can regulate cell apoptosis and autophagy $(13,14)$, and metabolic enzymes can also act as cellular RNA binding proteins to participate in the posttranscriptional control of specific mRNAs $(15,16)$. Finally, metabolites can be directly used as signal molecules to affect pro- and anti-inflammatory results $(17,18)$.

In the past few decades, people have been developing tools to detect changes in cellular metabolism. Traditional bulk metabolism research methods include extracellular flux analysis (EFA), steady-state metabolomics, etc. With the advent of single-cell applications, researchers can now estimate the metabolic status of individual immune cells in clinical samples. Technological advancements in measuring metabolites and metabolic activity have provided researchers with more tools than ever to understand and test the complexity of immunometabolism. Here is a brief introduction to the leading techniques used to study immunometabolism and its developmental history.

The EFA technique was initially used to study the effects of drugs in vitro by measuring cellular respiration and acidification. Researchers have developed various tools for its development, but the efficiency is still low, and the application is relatively limited (19). Since introducing the Seahorse Bioscience XFe Extracellular Flux Analyzer in 2009, it has brought innovation to this technology. It is the first in the world to use 24 -well or 96-well microwell plates as a platform, which can record the extracellular acidification rate (ECAR) and oxygen consumption rate (OCR) in real-time as key metabolic readings and provide indirect measurements of glycolysis and mitochondria respiration (20). It dramatically expands the research horizon of cell metabolism and extends it to the field of immune cells. It provides tools for exploring the upstream biology and cellular and molecular mechanisms that control immune cell responses through realtime metabolic analysis of live cells. Since 2012, there have been studies using this method to study the metabolic changes of dendritic cells (21), T cells (22), monocytes (23), and macrophages (24). However, it cannot provide detailed information about the metabolic pathways other than glycolysis and the TCA cycle in the mitochondrial activity. It also cannot capture a single cell's metabolism information.

Flow cytometry (FCM) is one of the most straightforward and informative single-cell methods for analyzing metabolic characteristics. In 1969, Van Dilla Fulwyler et al. invented the first fluorescence detection cytometer to quickly count human white blood cells (25). With the monoclonal antibody technology proposed by Kochler and Milstein in 1975 (26), the combination of cytometer and immunity and continuous development have gradually formed the current flow cytometer. It is no longer only used for cell counting but quantifies proteins, nucleic acids, and metabolites. Since this technique has been developed for many years, commercially metabolite fluorescent dyes and analogs are abundant and easy to use. Immune cells incubated with a fluorescently labeled substrate will occupy the substrate with 


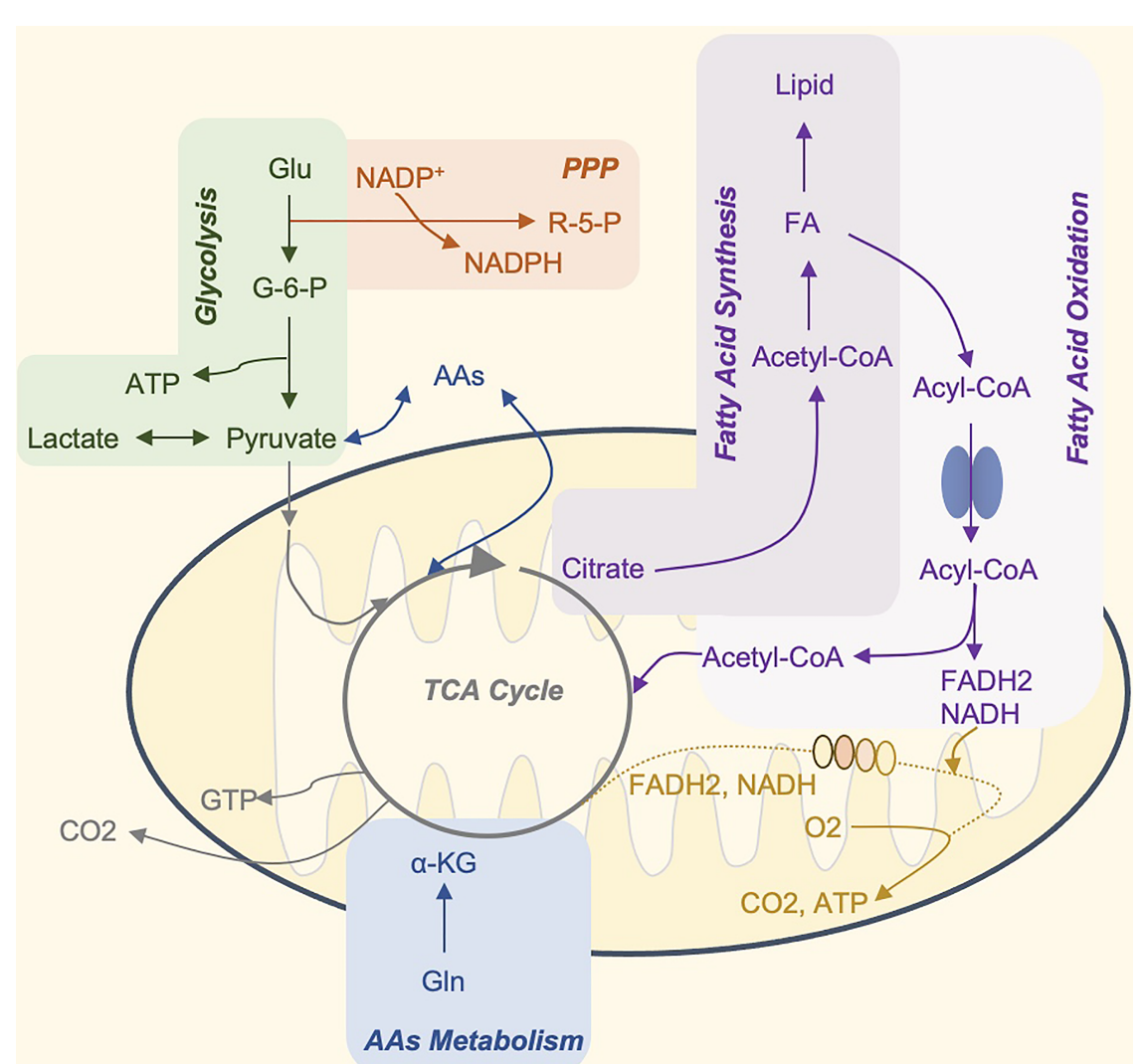

FIGURE 1 | The Core Metabolic Pathways of Immune Cells. Glucose is the primary energy supply substance. It can produce ATP through glycolysis, or tricarboxylic acid cycle, and oxidative phosphorylation. In addition, glucose 6-phosphate, the product of the first step of glycolysis, can participate in the production of pentose phosphate and NADPH by PPP, and participate in nucleotide metabolism and glutathione reduction, respectively (not shown in the figure). The TCA cycle is the hub of sugar, fat, and amino acid metabolism, and the three major nutrients can be transformed into each other through this cycle. Citrate can be used as a carrier to transport acetyl-CoA to the cytoplasm. Acetyl-CoA is the raw material for the synthesis of fatty acids and cholesterol. Many amino acids can be synthesized from intermediate products of the TCA cycle, and amino acids and fatty acids can also be used as alternative substrates for the process. For example, acetyl-CoA produced by $\beta$-oxidation of fatty acids and $\alpha$-ketoglutarate produced by the deamination of glutamate, can enter the TCA cycle and drive OXPHOS. Glu, glucose; G6P, glucose 6-phosphate; OXPHOS, oxidative phosphorylation; ATP, adenosine triphosphate; GTP, guanosine triphosphate; PPP, pentose phosphate pathway; AAs, amino acid synthesis; FA, fatty acids; R-5-P, ribose 5-phosphate; NADPH, reduced form of nicotinamide adenine dinucleotide phosphate; NADH, nicotinamide adenine dinucleotide FADH2, reduced flavine adenine dinucleotide.

an endogenous transporter. After washing the exogenous matrix, flow cytometry can quantify the uptake of metabolites from the matrix by the cells. The additional advantage of these techniques is that they required only a small number of cells, making flow cytometry a popular choice for rapidly verifying differences in metabolic activity $(27,28)$.

Cytometry + time of flight (CyTOF) is a flow cytometry technique based on heavy metal isotope-labeled antibodies. The key feature is that it no longer needs to use fluorescein-labeled antibodies like traditional flow cytometry. Since the sample will enter the mass spectrometer for analysis, it is also called mass spectrometry flow cytometry $(29,30)$. The application of this technique, on the one hand, has dramatically increased the number of flow detection channels to hundreds, increasing the amount of information obtained from a single sample. On the other hand, because the detection overlaps and the background are very low in cell analysis (since heavy metals will not naturally appear in the cell), avoiding signal interference between channels and improving data reliability. CyTOF is an excellent tool for tumor immunology. It can be used to phenotype tumor infiltrating immune cells and understand the metabolic spectrum of immune cells in the tumor microenvironment (31). CyTOF is also used to analyze histone acetylation markers and cell lineage markers simultaneously, thereby enabling the detection of histone acetylation changes in samples (32). Therefore, CyTOF that combines acetylation markers, metabolic signals, and lineage markers can finally reveal many new connections between metabolism and epigenetic regulation in complex immunocytes.

Although combined with mass spectrometry, the label's quantity of detectable metabolites is limited because CyTOF is an antibody labeling-based technique. Moreover, the three metabolic detection techniques mentioned above are all indirect, which cannot directly measure the metabolites' 
content in cells. So, is there a way to directly measure the level of metabolites? The answer is metabolomics.

Metabolomics is a new emerging omics after genomics, proteomics, and transcriptomics. Since 1998, related research has increased rapidly, but it still lags far behind genomics and proteomics (33). For a given time point, metabolomics analysis can measure the steady-state levels of a series of metabolites through a liquid or gas chromatography-mass spectrometry (LC-MS or GC-MS) and nuclear magnetic resonance (NMR) (34-36). Most MS-based methods still require high levels of input flux (approximately 100,000 s of cells). With technology development, single-cell metabolomics tools have been gradually developed and applied (37). Representative single-cell metabolism mass spectrometry tools include Matrix-Assisted Laser Desorption Ionization Time of Flight (MALDI-TOF) $(38,39)$, Secondary Ion Mass Spectrometry (SIMS) (40), Direct Infusion Mass Spectrometry (DI-MS) (41), Capillary Electrophoresis Mass Spectrometry (CE-MS) (42), etc. Metabolomics analysis of single-cell expands its applications. Metabolomics can detect metabolite content in all metabolic pathways, identify changed metabolite levels, and provide information for studying the role of metabolites in regulating cell function (43). Metabolomics analysis can be divided into untargeted and targeted metabolomics. Untargeted metabolomics analysis can detect as many metabolites as possible, but it requires expertise in metabolite identification, and its sensitivity may also be limited. In other words, although thousands of signals can be detected, only a few substances can be identified. However, this can lead to discoveries and is very suitable for the development of biomarkers. Targeted metabolomics evaluates pre-selected metabolites, generates data with higher sensitivity, and allows precise absolute quantification of metabolite concentrations, facilitating the analysis of known changes in metabolite levels $(44,45)$.

Nowadays, we can use various metabolic detection techniques to observe the processes of metabolic remodeling in different immune cell subsets. These techniques have their advantages and disadvantages (Table 1). However, the raw data obtained by these techniques are either indirect or covered limited. In order to promote the progress of these techniques and the next generation of immunometabolism research, there are two main directions: 1) Improve the depth and quality of data analysis of single-cell level analysis; 2) Improve ability to analyze the temporal and spatial distribution of metabolism.

Based on the above detection techniques, researchers have promoted the understanding of immunometabolic reprogramming. Next, this review discusses the function of immune cells at the maternal-fetal interface and their metabolic preferences required to perform this function explores the potential role of immune cell metabolic reprogramming in the maternal-fetal immune interface.

\section{THE METABOLIC REPROGRAMMING OF THE NK CELLS IN THE ENDOMETRIUM AND DECIDUA}

NK cells are the most abundant immune cells in the endometrium during embryo implantation and placenta formation. In early pregnancy, decidual NK cells (dNK cells) account for about $50 \%$ to $90 \%$ of decidual lymphocytes, and it gradually decreases during the middle and late pregnancy (51). They play a crucial role in the uterine immunity to promote blood vessel remodeling at the maternal-fetal interface, maintain the stability of the immune microenvironment, enable the healthy growth of the fetus, and protect the mother from harmful pathogens through appropriate inflammation $(52,53)$.

Decidual NK cells are mainly $\mathrm{CD} 56^{\text {bright }} \mathrm{CD} 16^{-} \mathrm{NK}$ cell subgroups. Unlike the cytotoxic $\mathrm{CD} 56^{\mathrm{dim}} \mathrm{CD} 16^{+} \mathrm{NK}$ cells in peripheral blood, it is a highly immunomodulatory cell (54)

TABLE 1 | Leading techniques for detection of immune cell metabolism and their advantages and disadvantages.

\begin{tabular}{|c|c|c|c|c|}
\hline Techniques & Advantages & Disadvantages & $\begin{array}{c}\text { Time of appearance } \\
\text { in immunology }\end{array}$ & $\begin{array}{c}\text { Representative } \\
\text { works }\end{array}$ \\
\hline $\begin{array}{l}\text { Extracel-lular flux } \\
\text { analysis }\end{array}$ & $\begin{array}{ll}\text { - } & \text { Simple } \\
\text { - } & \text { Affordable } \\
\text { - } & \text { Fast }\end{array}$ & $\begin{array}{l}\text { - Unable to detect single cell metabolism } \\
\text { - Only the information of glycolysis and TCA cycle in } \\
\text { mitochondria can be obtained } \\
\text { - Indirect detection }\end{array}$ & $1970 s$ & $\begin{array}{l}\text { Katherine et al. } \\
2016(46)\end{array}$ \\
\hline Flow cytometry & $\begin{array}{l}\text { - } \text { Common laboratory instruments } \\
\text { - } \text { Affordable } \\
\text { - Fast }\end{array}$ & $\begin{array}{l}\text { - Indirect detection } \\
\text { - Low throughput } \\
\text { - Fluorescence may cause interference to the } \\
\text { background }\end{array}$ & $1980 s$ & $\begin{array}{l}\text { Argüello et al. } \\
2020(47)\end{array}$ \\
\hline CyTOF & $\begin{array}{ll}\text { - } & \text { High throughput } \\
\text { - } & \text { No interference between channels } \\
\text { - } & \text { Fast }\end{array}$ & $\begin{array}{ll}\text { - } & \text { Indirect detection } \\
\text { - } & \text { Expensive equipment }\end{array}$ & $2010 s$ & $\begin{array}{l}\text { Horowitz et al. } \\
2013 \text { (48) }\end{array}$ \\
\hline $\begin{array}{l}\text { Steady state } \\
\text { metabolo-mics }\end{array}$ & $\begin{array}{l}\text { - Direct detection } \\
\text { It can target or untargeted detect } \\
\text { the entire metabolome }\end{array}$ & $\begin{array}{l}\text { - } \quad \text { Expensive equipment } \\
\text { Metabolome is unstable and easily affected by } \\
\text { storage conditions } \\
\text { - High-level technical personnel need }\end{array}$ & $2010 s$ & $\begin{array}{l}\text { Tannahill et al. } \\
2013 \text { (49) }\end{array}$ \\
\hline $\begin{array}{l}\text { Single cell } \\
\text { metabolo-mics }\end{array}$ & $\begin{array}{l}\text { - Direct detection } \\
\text { - It can target or untargeted detect } \\
\text { the entire metabolome }\end{array}$ & $\begin{array}{l}\text { - } \quad \text { Expensive equipment } \\
\text { - } \quad \text { High-level technical personnel need } \\
\text { - Low abundance and low ionization efficiency } \\
\text { metabolites cannot be detected }\end{array}$ & 2010s & $\begin{array}{l}\text { Hartmann et al. } \\
2021(50)\end{array}$ \\
\hline
\end{tabular}


that can secrete cytokines such as interferon- $\gamma($ IFN- $\gamma)$ promotes angiogenesis and tissue remodeling (55). The function of $\mathrm{dNK}$ cells in early pregnancy is regulated by the expression of trophoblast human leucocyte antigen-I (HLA-I) (such as HLA$\mathrm{C} / \mathrm{G} / \mathrm{E}$ ) and the interaction of inhibitory or activating receptors (such as KIR, CD94/NKG2, ILT family) with trophoblast (56). A large amount of evidence indicates that the specific combination of highly polymorphic killer cell immunoglobulin-like receptors (KIR) on dNK cells and HLA-C on invasive trophoblasts affect decidual vascular recasting and thus affects the success of pregnancy (57).

The metabolic regulation of NK cells is driven by complex molecular mechanisms, among which the mammalian target of rapamycin (mTOR) signaling pathway is the most important and the most widely studied in metabolic regulation (Figure 2). During NK cell activation, glycolysis and mitochondrial function are enhanced via the activation of mTOR. The pharmacological inhibition of $\mathrm{mTOR}$ by rapamycin reduces the up-regulation of IL-2/IL-12-stimulated glycolysis in mouse
NK cells and decreases IL-2-stimulated levels of human NK cell glycolysis $(58,59)$. Compared with CD $56^{\mathrm{dim}} \mathrm{NK}$ cells, CD56 ${ }^{\text {bright }}$ NK cells show a more robust metabolic response to IL-2 or IL12/IL-15 stimulation and have higher mTOR activity. It preferentially upregulates the nutrient receptors $\mathrm{CD} 71$ and CD98 in an mTOR-dependent manner, expressing higher levels of glucose transporter 1 (GLUT1) to absorb glucose quickly (60). However, excessive activation of mTOR may cause mitochondrial fragmentation, thereby impairing mitochondrial function (61).

Although lipid synthesis is essential for the metabolic reprogramming of $\mathrm{T}$ cells, inhibition of the lipid synthesis pathway has the most negligible impact on NK cell effector function and proliferation $(62,63)$. It is still unknown whether fatty acids (FA) can provide energy for NK cells as an essential nutrient. FA administration, and peroxisome proliferatoractivated receptor (PPAR) agonists, inhibited mTOR-mediated glycolysis and inhibited NK cell effector function and metabolism (64). Metabolic activation is essential for NK cells

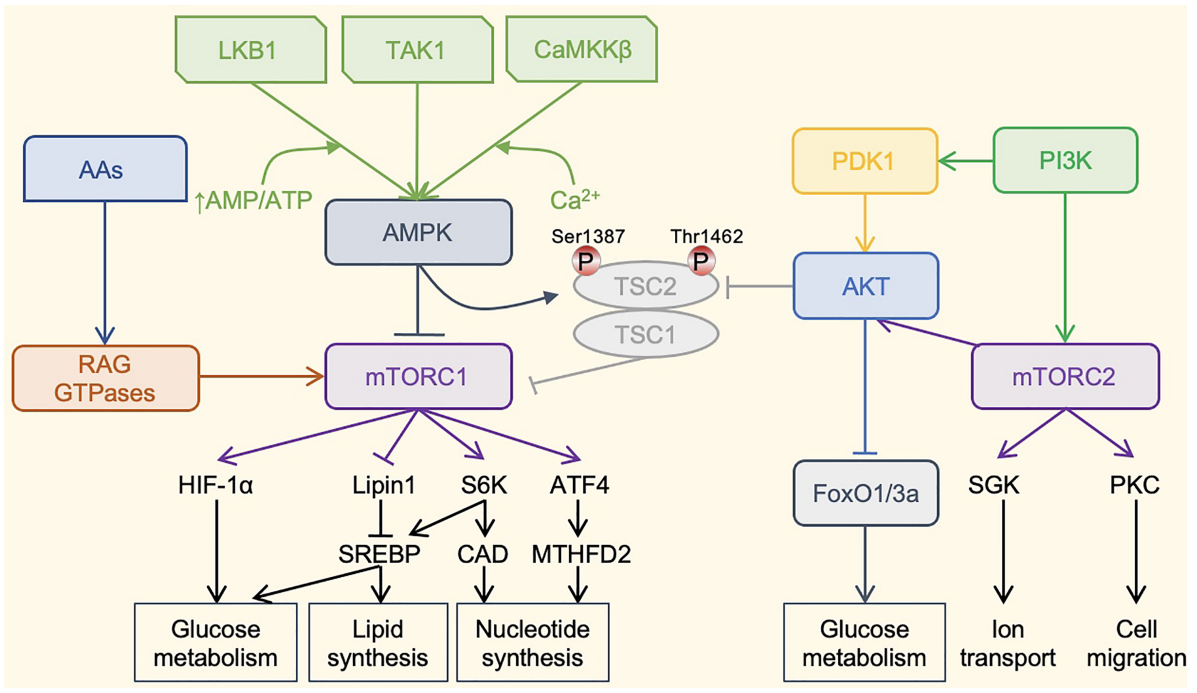

FIGURE 2 | The mTOR-centered Metabolic Regulatory Signaling Pathway. mTOR is a crucial eukaryotic cell regulator, which affects the expression of cytokines in cells, participates in immunosuppression, and regulates cell growth. mTOR contains two complexes. mTORC1 promotes cell growth by promoting glucose metabolism. It can enhance the translation of the transcription factor HIF-1 $\alpha$, thereby promoting glucose metabolism. It can also activate the transcription factor SREBP to promote lipid synthesis and meet the formation of membranes during cell growth. mTORC1 also promotes the synthesis of nucleotides required for DNA replication and ribosome synthesis during cell growth and proliferation. mTORC1 promotes the expression of ATF4, further increases the expression of MTHFD2, and provides carbon units for purine synthesis. Phosphorylation of S6K1 can activate the CAD enzyme and promote pyrimidine synthesis. mTORC1 is mainly regulated by upstream AMPK. AMPKa is activated by phosphorylation by LKB1, TAK1, or CaMKKb kinase. LKB1 promotes the enhancement of AMPK phosphorylation under a high AMP/ATP ratio. Activated AMPK can cause AMPK-dependent phosphorylation of Ser 1387 of TSC2 and reduce mTORC1 activation. AMPK can also directly phosphorylate Raptor to reduce the activity of mTORC1. The TSC1-TSC2 complex also integrates the PI3K-Akt pathway, which regulates the activity of mTORC1 through the AKT-dependent phosphorylation of Thr1462. In addition, amino acids can activate mTORC1 through the RAG family of small GTPases. The most important role of mTORC2 may be the phosphorylation and activation of Akt. Akt is a key effector of insulin/PI3K signaling. Once activated, Akt promotes cell survival, proliferation and growth through phosphorylation and inhibition of several key substrates, including FoxO1/3a transcription factor and TSC2. Finally, mTORC2 also phosphorylates and activates SGK1 and PKC, and regulates ion transport and cell migration. PI3K can directly activate mTORC2 through phosphorylation. mTOR, mammalian target of rapamycin; HIF-1 $\alpha$, hypoxia-inducible factor-1 $\alpha$; SREBP, Sterol regulatory element binding proteins; ATF4, activating transcription factor 4; MTHFD2, methylenetetrahydrofolate dehydrogenase 2; S6K1, ribosomal protein S6 kinase 1, CAD, carbamoyl-phosphate synthetase 2, aspartate transcarbamoylase, dihydroorotase; AMPK, adenosine 5'-monophosphate-activated protein kinase; LKB1, liver kinase B1; TAK1, transforming growth factor beta-activated kinase 1; CaMKKb, $\mathrm{Ca}^{2+}$ /calmodulin-dependent protein kinase kinase beta; TSC, tuberous sclerosis complex; PI3K, phosphoinositide 3-kinase; Akt, serine-threonine kinase; PDK1, phosphoinositide dependent kinase-1; RAG GTPases, ras-related guanosine 5'-triphosphate-binding protein; FoxO1/3a, forkhead box protein 01/3a; SGK1, serum/glucocorticoid-regulated kinase 1; PKC, protein kinase C; Thr1462, threonine 1462 ; Ser 1387 , serine 1387. 
to produce effector proteins (including IFN- $\gamma$ and Granzyme B) and to form correct immune synapses with target cells $(59,64)$.

Glutamine $(\mathrm{Gln})$ is another crucial substrate for cell metabolism; it enters the TCA cycle but does not sustain oxidative phosphorylation (OXPHOS) in activated NK cells. It plays an essential role for amino acid-controlled c-Myc for NK cell metabolism and function (65). Gln helps absorb the amino acids necessary for the high rate of $\mathrm{c}-\mathrm{Myc}$ protein synthesis through the L-type amino acid transporter SLC7A5, counteracting the continuous degradation of c-Myc, while Gln withdrawal results in the loss of c-Myc protein. c-Myc controls the expression of metabolic mechanisms, including glucose transporters and glycolytic enzymes, which are necessary to support increased metabolism during NK cell activation (65).

However, the current research mainly focuses on peripheral blood NK cells, and the research on $\mathrm{dNK}$ cell metabolism is relatively limited. Since dNK cells are the most abundant immune cells in the maternal-fetal interface, studying their metabolic reprogramming is likely to yield breakthrough achievements.

\section{METABOLIC REPROGRAMMING OF MACROPHAGES AFFECTS POLARIZATION AND PLAYS A ROLE IN THE MATERNAL-FETAL INTERFACE}

Macrophages are the second largest immune cells population in the endometrium and decidua after NK cells, accounting for 20$30 \%$ of lymphocytes (66). Generally, macrophages are classified into pro-inflammatory M1 macrophages and anti-inflammatory M2 macrophages based on their activation status (67). The "plasticity" of macrophages allows them to respond rapidly to the immune response triggered by embryonic implantation, which plays a pivotal role in immune regulation and promotes angiogenesis (4). During pregnancy, the number and ratio of M1/M2 macrophages at the maternal-fetal interface change dynamically: In the early stage of embryo implantation, decidual macrophages are dominated by M1 macrophages, and then, metabolic reprogramming occurs, more macrophages are polarized into M2 macrophages, which prevents rejection of the fetus. Until late pregnancy, the polarization of macrophages gradually tends to change to inflammation, which is endogenously prepared for delivery $(68,69)$. The dynamic balance between M1 and M2 macrophages is essential for the whole process of normal pregnancy (70). Inappropriate macrophage polarization, whenever it occurs, is usually associated with abnormal pregnancy, such as spontaneous abortion (71), preterm delivery (PTL) (72), preeclampsia (PE) (73), fetal growth restriction (FGR) (74).

Macrophages with different polarization states have apparent differences in glycometabolism pathways. Pro-inflammatory M1 macrophages are mainly powered by anaerobic glycolysis, providing instantaneous and rapid energy to eliminate microorganisms that invade the body, while mitochondrial OXPHOS continuous generates energy for anti-inflammatory
M2 macrophages (75). Studies have shown that a normal mitochondrial function is required for the polarization/ repolarization of macrophages to M2 macrophages (76). When lipopolysaccharide (LPS) and IFN- $\gamma$ are stimulated, the mitochondrial function of macrophages is impaired or inhibited, the level of OXPHOS is reduced, and anaerobic glycolysis has increased the polarization of macrophages to M1 type. Decreased OXPHOS leads to the accumulation of TCA cycle metabolites such as succinate, and upregulates hypoxiainducible factor- $1 \alpha$ (HIF-1 $\alpha$ ) (49). HIF- $1 \alpha$ can induce the transcription of related transcription factors, such as nuclear factor $\kappa \mathrm{B}(\mathrm{NF}-\kappa \mathrm{B})$, and positively regulate related key enzymes (such as hexokinase) and transporters (such as GLUT1) to promote metabolic reprogramming of anaerobic glycolysis and PPP, thereby affecting the polarization and biological functions of macrophages (5).

In recent years, many researchers have tried to use glycometabolism reprogramming to regulate the polarization of macrophages and change their functions as a means of disease prevention and treatment. Wenes $\mathrm{M}$ et al. have reported that blocking the regulation in development and DNA damage responses 1 (REDD1) of macrophages can change their metabolism and stimulate their glycolysis. Promote macrophages and tumor cells to start "glucose competition", so that a common and robust blood vessel barrier is formed around the tumor, blocking the metastasis of cancer cells (77). This suggests that glycometabolism reprogramming can change the polarization state of macrophages and affect their related biological functions. Van den Bossche $J$ et al. have also found that restoring mitochondrial function through inducible nitric oxide synthase (iNOS) inhibition may help improve the reprogramming of $\mathrm{M} 1$ to $\mathrm{M} 2$, thereby controlling inflammation (76). Herein, glycometabolism reprogramming to change the function of macrophages may be used as a therapeutic target for diseases.

About lipid metabolism, researchers have observed different levels of arachidonic acid mobilization and other FA changes in different differentiated types of macrophages, which indicates that the lipidome can reflect the process of cell membrane remodeling and macrophage polarization (78). In the polarization of M2 macrophages stimulated by IL-4, FAs absorption and FAO levels increased significantly. This is mainly due to the activation of the transcription factor recombinant signal transducer and activator of transcription 6 (STAT6) by IL-4, which in turn induces the synthesis of peroxisome proliferative actives receptor $-\gamma$ coactivator- $1 \beta$ (PGC-1 $\beta$ ) (79). At the transcriptional level, peroxisome proliferator-activated receptor $\gamma$ $(\mathrm{PPAR} \gamma)$ is a key transcriptional regulator of macrophage mitochondrial function and FAO, and can enhance alternative activation $(49,80)$. Lipids can also regulate protein functions by acting as remote signaling molecules. For example, the enhanced esterification of cholesterol acyl transferase 1 (ACAT1) induces more free cholesterol accumulation, and causes the up-regulation of inflammatory signals like toll-like receptors (TLR) and NF- $\kappa B$ signals through lipid rafts (5). This signal is also a key factor in the phosphatidylinositol 3-kinase (PI3K)/serine-threonine kinase 
(Akt) signal pathway. Its activation can lead to increased polarization and migration of M1 macrophages and promote the secretion of inflammatory cytokines (81).

OXPHOS and amino acid metabolism are also closely related to $\mathrm{M} 2$ polarization and anti-inflammatory properties. Inhibiting the level of OXPHOS can reduce the expression level of arginase 1 , which is harmful to tissue repair and remodeling (82). Gln deprivation also reduces the polarization of M2 (83). Gln, like glucose, is considered an important energy source for immune cells. Xiao W et al. have reported that Gln supplementation in the diet of rats can reverse the impaired function of macrophages to some extent (84). On the contrary, inhibition of glutamine synthetase can promote the metabolic reprogramming of M2 macrophages to polarize to M1 and promote inflammation.

In conclusion, the dynamic homeostasis and function of macrophages during pregnancy are influenced by various metabolites in the body, and in turn, their metabolic reprogramming plays a crucial regulatory role in maintaining the microenvironment at the maternal-fetal interface.

\section{THE INFLUENCE OF T CELL METABOLISM REPROGRAMMING ON THE ESTABLISHMENT OF THE MATERNAL-FETAL INTERFACE}

There are many subsets of $\mathrm{T}$ cells, which play different roles in establishing and maintaining pregnancy. Decidual T cells account for about $10 \%$ to $20 \%$ of decidual lymphocytes (85). Compared with peripheral blood, the proportion of $\mathrm{CD}^{+}$and $\mathrm{CD}^{+} \mathrm{T}$ cells in decidua is inverted, with $\mathrm{CD}^{+} \mathrm{T}$ cells predominantly (45\% 75\%) (86). As the pregnancy progresses, the number of $\mathrm{T}$ cells in the maternal-fetal interface gradually increases. $\mathrm{T}$ cells become the dominant lymphocyte in late pregnancy, accounting for about $60 \%$ of the decidual lymphocytes (87). $\mathrm{CD}^{+} \mathrm{T}$ cell subsets include helper T cells (Th) and regulatory T cells (Treg), and Th cells can be divided into Th1, Th2 and Th17 $(88,89)$. They regulate immunity by secreting different cytokines.

Th1/Th2 cell-mediated immune balance is one of the primary mechanisms for maintaining maternal-fetal immune tolerance. Under normal pregnancy conditions, the Th1/Th2 balance is biased towards Th2, because Th2-type cytokines (IL-4, IL-5, IL-9 and IL-13) can inhibit inflammation, reduce tissue damage, and stimulate growth and invasion of trophoblast cells, and increase uterine receptivity (86). Once this Th2-type immunodominance is broken, the incidence of spontaneous abortion increases significantly (90).

Th17/Treg is another independent cytokine network following Th1/Th2. It has been reported that both Th17 and Treg are differentiated from initial $\mathrm{CD}^{+} \mathrm{T}$ cells in a transforming growth factor- $\beta$ (TGF- $\beta$ ) concentration-dependent manner, driven by high concentrations of TGF- $\beta$ initial $\mathrm{CD}^{+}{ }^{+} \mathrm{T}$ cells differentiate into Tregs, and at low concentrations differentiate into Th17 (91). It has also been reported that decidual Treg cells are selectively recruited from the peripheral blood to the maternal-fetal interface through the action of chemokines CCL2 (92), CCL19 (93), CXCL12 (94), and chemokine receptors CCR5 (95), CCR7 (96) and CXCR4 (94). Treg cells in the maternal-fetal interface of normal pregnancy are significantly higher than those in the peripheral blood and the decidua of patients with spontaneous abortion (97). The Th17 cells and IL-17 secreted by Treg cells jointly promote the proliferation of trophoblast cells and invade the decidual tissue, which plays a positive role in the immune microenvironment of the maternalfetal interface (98). The abundant Treg at the maternal-fetal interface in the early pregnancy also inhibits the hyperproliferation of Th17 and reduces the production of IL-17 through paracrine or direct contact. Once the number of Treg cells is reduced, or the function is impaired, the excessive enrichment of Th17 will cause excessive inflammation, which will cause a variety of adverse pregnancy outcomes, such as unexplained recurrent spontaneous abortion (URSA) and PE $(99,100)$.

Decidual $\mathrm{CD}^{+} \mathrm{T}$ cells also play an essential role in establishing the maternal-fetal interface (87). The function of $\mathrm{CD} 8^{+} \mathrm{T}$ cells in the endometrium of ordinary non-pregnant women is similar to that of peripheral blood $\mathrm{CD} 8^{+} \mathrm{T}$ cells. It has potent cytotoxicity and exerts immune protection function by releasing cytotoxic particles. During pregnancy, decidual $\mathrm{CD}^{+} \mathrm{T}$ cells exhibit temporary dysfunction, with high expression of IFN- $\gamma$, low expression of perforin and granzyme B $(101,102)$. Trophoblasts can promote the expression of CTLA- 4 and Tim- 3 molecules on decidual $\mathrm{CD}^{+} \mathrm{T}$ cells in an HLA-C/G-dependent manner. Decidual $\mathrm{CD}^{+} \mathrm{T}$ cells co-expressing Tim-3, CTLA-4, or PD-1 are highly proliferative, can secrete large amounts of Th2 cytokines and play an active role in maintaining maternal-fetal tolerance (102-104).

$\mathrm{T}$ cells display different metabolic profiles according to their activation status. Naive $\mathrm{T}$ cells mainly rely on OXPHOS to maintain a resting state, while activated $\mathrm{T}$ cells maintain their growth by switching to glucose and lipid metabolism. The traditional view is that the aerobic glycolysis rate of proliferating cells is very high, even if there is enough oxygen to support OXPHOS. This phenomenon is called the Warburg effect (105). Substrate-level phosphorylation produces ATP during glycolysis, pyruvate is converted to lactate, and biological macromolecules are synthesized for cell growth and division (106). These metabolic changes can be directly triggered by $\mathrm{T}$ cell receptors (TCR). In naive $\mathrm{T}$ cells, costimulatory molecules CD28 cooperate with the TCR of antigen-presenting cells (APC) to upregulate the membrane expression of the GLUT1 through the mTOR pathway to promote glucose absorption $(107,108)$. These effects can be offset by immunosuppressive molecules, including CTLA4 and PD-1, which prevent the immune response by inhibiting glucose uptake and promoting endogenous fatty acid oxidation (109).

Th1, Th2 and Th17 show strong glycolysis preferences in mitochondrial metabolism, while Treg cells exhibit mixed metabolism involving glycolysis, lipid oxidation, and OXPHOS (110). In particular, Th17 cells have an increased dependence on glycolysis. HIF- $1 \alpha$, as an oxygen-sensitive transcription factor, can regulate the expression of glycolysis genes in Th17 cells (111, 112). Blocking glycolysis will reduce the differentiation of TH17 
cells, which facilitates the formation of Tregs (111). In addition, studies have shown that extracellular salts (like $\mathrm{NaCl})(113,114)$ and short-chain FA (115) affect the homeostasis of Th17 and Treg. This suggests that the metabolic microenvironment (i.e., nutrient and oxygen availability) may affect $\mathrm{T}$ cell polarization.

The differentiation of Th2 cells depends on the mTOR-mediated metabolic transition from OXPHOS to aerobic glycolysis, but the complete role of metabolism in Th2 polarization is not fully understood. Reprogramming of glycolysis and OXPHOS in Th2 cells depends on the activity of the small GTPase RhoA $(46,116)$. mTORC2 regulates RhoA activity and is essential for the production of Th2 cells (117). For the Th1 cell phenotype, mTORC1 may be essential (117). The transcription factor interferon regulatory factor 4 (IRF4) is also essential in the glycolytic reprogramming and differentiation of Th1 cells and can prevent the expression of glycolytic enzymes by regulating the transcription suppressor B-cell lymphoma 6 (BCL-6) (118).

The oxidation of free fatty acids (FFA) produces acetyl-CoA, which can be further metabolized in the TCA cycle, and FADH2 and NADH, which can be directly used by the electron transport chain (ETC) to produce ATP. Metformin is a metabolic stress factor that can activate the adenosine monophosphate-activated protein kinase (AMPK) energy sensor. It can enhance the production of $\mathrm{CD}^{+} \mathrm{T}$ cells (119). After AMPK is activated, it inhibits mTORC1 activity in response to energy stress (120). Consistent with this, rapamycin also inhibits mTORC1 and can enhance the production of $\mathrm{CD}^{+} \mathrm{T}$ cells $(121,122)$. On the other hand, mTORC2 is involved in FAO and stabilizes forkhead box protein $\mathrm{O} 1$ (FoxO1) through the Akt pathway to regulate the memory differentiation of $\mathrm{CD}^{+} \mathrm{T}$ cells $(123,124)$.

Treg cells depend on glycolysis for their differentiation and migration $(125,126)$. Glutamine or arginine can regulate the proliferation and activation of $\mathrm{T}$ cells and the expression level of Treg cell-specific transcription factor FoxP3 $(107,127)$.

These together reveal that a broad understanding of metabolic processes is emerging, which may help explain how metabolism determines the differentiation and function of Th1, Th2, Th17, Treg, and $\mathrm{CD}^{+} \mathrm{T}$ cells.

\section{THE INFLUENCE OF OTHER IMMUNE CELLS METABOLISM REPROGRAMMING ON THE ESTABLISHMENT OF THE MATERNAL-FETAL INTERFACE}

In addition to the above three types of immune cells, other immune cells with a smaller number also play an essential role in forming the maternal-fetal interface, such as dendritic cells (DC) and mast cells (MC).

DCs interact closely with other immune components and the endocrine system to maintain a pregnancy-friendly environment (128). As the most effective antigen-presenting cells in the immune system, DCs can regulate the differentiation of $\mathrm{T}$ cells $(129,130)$. In addition, they can inhibit the proliferation of NK cells (131) and may affect the polarization of macrophages (132).
Abnormal DC activity is related to various pregnancy-related diseases, such as RIF (133), preterm birth (PTB) (134), PE (135), and perinatal cardiomyopathy (PPCM) (136), etc.

DCs are mainly divided into four subgroups, including conventional DCs (cDCs), plasmacytoid DCs (pDCs), Langerhans cells (LCs), and inflammatory monocyte-derived DCs (infDCs) (137). However, studies on immune changes in pregnancy mainly focus on $\mathrm{CDC}$ and $\mathrm{pDC}$. Most current studies have shown that the $\mathrm{cDC} / \mathrm{pDC}$ ratio of pregnant women is higher than that of non-pregnant women $(134,138)$, indicating that $\mathrm{CDC}$ may play an important role in the process of maternalfetal tolerance. According to its maturity, DC can be divided into immature DC (imDC) and mature DC (mDC).

Decidual dendritic cells (dDCs) are a tolerant, immunomodulatory, and heterogeneous population, different from pDCs. dDCs are mainly immature DCs that express a combination of CD11c, HLA-G, CD14 and DC-SIGN (dendritic cell-specific ICAM-grabbing non integrin, CD209) (139). Observational studies have found that a higher level of mature peripheral or decidual DC is positively correlated with the development of adverse pregnancy (such as PE and abortion) (140, 141). This means that a delicate balance needs to be achieved between mature and immature $\mathrm{dDC}$ to ensure a successful pregnancy.

Metabolic processes have precise effects on DC functions, and manipulating these pathways can significantly change DC functions in specific ways. Due to the scarcity of DCs in vivo, in vitro experimental models are mainly used to study DC metabolism microenvironment.

A common phenomenon in immune cell activation, including DC, is the transition from catabolism, which is characterized by fatty acid oxidation and mitochondrial respiration, to anabolism. After immune cell activation, glycolysis is increased, and oxidative phosphorylation is decreased (142).

Under the stimulation of LPS, DCs can regulate mTOR signal, stabilize HIF1 $\alpha$ and increase iNOS expression, induce the synthesis of endogenous NO. This process induces the synthesis of endogenous $\mathrm{NO}$ and inhibits the electron transport chain, thereby inhibiting mitochondrial oxidative phosphorylation, which increases cellular glycolysis to maintain intracellular ATP levels $(143,144)$. In fact, in addition to glucose, several amino acids also affect the mTORC1/HIF1 $\alpha /$ iNOS signal, including leucine and glutamine, which are essential for mTORC1 activity, and arginine, which is the fuel for NO production on which iNOS relies (145). However, there are signs that once cells interact with $\mathrm{T}$ cells, the role of glucose in DCs initiating $\mathrm{T}$ cell responses will change. Activated T cells significantly increase the glucose and amino acid uptake rate, and the immediate microenvironment surrounding $\mathrm{DC}$ in the $\mathrm{T}$ cell cluster becomes deficient in nutrients. This competition for glucose or amino acids results in a prolonged $\mathrm{T}$ cell response (143).

The imDCs mainly rely on FAO for energy, which makes them have a longer lifespan (144). $\omega 3$ polyunsaturated fatty acids can inhibit the expression of the immune phenotype of DCs and the release of cytokines in vitro, and reduce their ability to stimulate $\mathrm{T}$ cell proliferation. The unsaturated fatty acid 
metabolite prostaglandin can regulate $\mathrm{mDCs}$, upregulating the expression of costimulatory molecules and pro-inflammatory cytokines at low concentrations while inhibiting the expression at higher concentrations (5). However, most of these data come from In vitro studies, the metabolic environment in which DC competes with nearby cells for nutrition is difficult to imitate in vitro, and it is also difficult to measure in vivo. However, it is undeniable that the metabolic reprogramming of DC is of vital importance to its function.

Mast cells also have a positive effect on embryo implantation (146). It can secrete hemcryptin-1 (a glycan-binding protein) to support the survival of trophoblasts, placenta and fetal growth. Mice lacking mast cells (including uterine mast cells) have impaired implantation (147). Uterine NKs and MCs can also balance their influence at the fetal-maternal interface and jointly promote SA remodeling and fetal position (148). However, no researchers have conducted metabolic reprogramming studies on MCs, so this review will not describe it a lot.

\section{SUMMARY AND OUTLOOK}

Immune homeostasis relies on immune cells to acquire appropriate functions by adaptively regulating their metabolic preferences to rebalance their immune microenvironment. This review summarized the metabolic phenotypes of the main immune cells in the context of maternal-fetal interface (Figure 3). Investigation on the metabolic reprogramming of immune cells at the maternal-

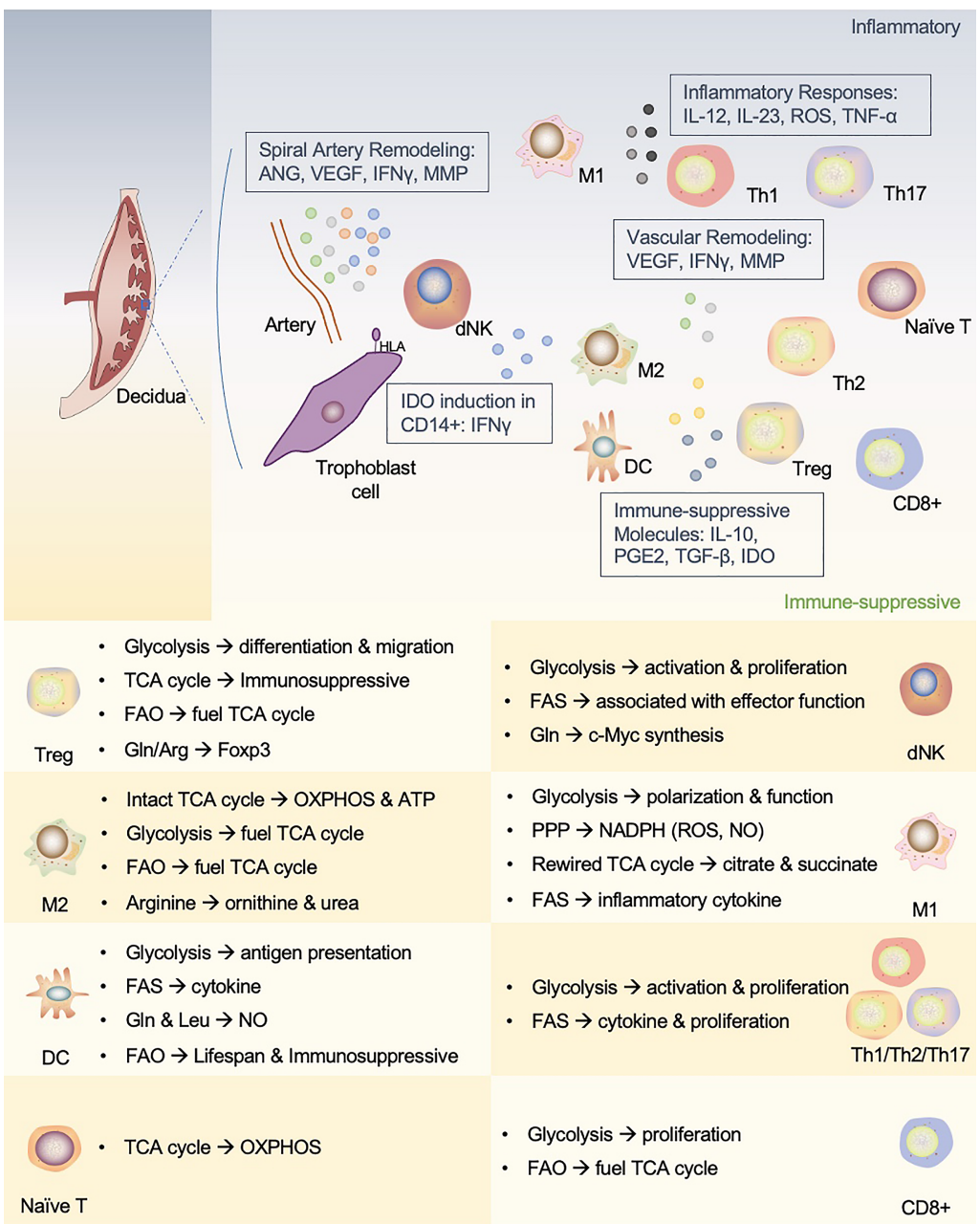

FIGURE 3 | An interactive network of immune cells and underlying metabolic phenotypes at the maternal-fetal interface. This figure proposes a unique immune microenvironment at the maternal-fetal interface and potential metabolic mechanisms that support phenotypic adaptation during pregnancy. In human maternal-fetal interface, trained NK cells promote inflammatory and pro-angiogenic cytokines that regulate angiogenesis and trophoblast invasion and migration. The release of IFN- $\gamma$ also upregulates IDO expression in antigen-presenting cells (APCs, such as macrophages and DCs), which influences T cell activation and facilitates Treg cell induction. In addition, APCs play a role in vascular remodeling in early pregnancy. dNK, decidual NK cells; M1, M1 macrophages; M2, M2 macrophages; Th1, T-helper 1 cells; Th2, T-helper 2 cells; Th17, T-helper 17 cells; Treg, regulatory T cells; DC, dendritic cell; ANG, angiotensin; VEGF, vascular endothelial growth factor; MMP, matrix metalloprotein; IDO, indoleamine2,3-dioxygenase; PGE2, prostaglandin E2. 
fetal interface can help us understand the mechanisms of pregnancy and provide insights into developing diagnostic and therapeutic approaches for pregnancy-related diseases. However, research on immunometabolism in pregnancy is still lagging due to clinical ethics and technological developments.

First of all, in most cases, researchers only focus on the most abundant cell groups found in the blood or bone marrow rather than tissue residents or recruited cells that actively participate in host defense and tissue homeostasis. Secondly, the current research does not consider the observed diversity of immune cell lineages or tissue-specific functions. They only consider the metabolic reprogramming of a single or a group of immune cells, ignored interactions between various immune cells in the tissue that may cause metabolic reprogramming again. Thirdly, although many inducers and sensors have been identified for activating immune cells, we lack an understanding of how these signals are integrated into cohesive metabolic programs that support cell effector functions. Last but not the least, it is technically challenging to identify the relative quantity of "all metabolites" in metabolomics. For example, GC-MS effectively quantifies organic acids (such as TCA cycle intermediates), but it is not practical for most glycolysis intermediates. Some volatile or unstable substances may not be detected by the LC-MS method, while GC-MS may be a better choice. Thus, multiple platforms need to be used to generate a complete metabolic data set.

As the cost of applying these new techniques decreases and the application becomes more comprehensive, more valuable, original breakthroughs can be made in this field. There are many questions urgently requiring in-depth investigation and deliberation regarding reproductive immunology. Such as which side plays

\section{REFERENCES}

1. Moffett ALoke C. Immunology of Placentation in Eutherian Mammals. Nat Rev Immunol (2006) 6:584-94. doi: 10.1038/nri1897

2. Cha JSun XDey SK. Mechanisms of Implantation: Strategies for Successful Pregnancy. Nat Med (2012) 18:1754-67. doi: 10.1038/nm.3012

3. PrabhuDas MBonney ECaron KDey SErlebacher AFazleabas A, et al. Immune Mechanisms at the Maternal-Fetal Interface: Perspectives and Challenges. Nat Immunol (2015) 16:328-34. doi: 10.1038/ni.3131

4. Newton ERMay L. Adaptation of Maternal-Fetal Physiology to Exercise in Pregnancy: The Basis of Guidelines for Physical Activity in Pregnancy. Clin Med Insights Womens Health (2017) 10. doi: 10.1177/1179562X17693224. $1179562 X 17693224$.

5. O’Neill LAJPearce EJ. Immunometabolism Governs Dendritic Cell and Macrophage Function. J Exp Med (2016) 213:15-23. doi: 10.1084/ jem. 20151570

6. Ip WKEHoshi NShouval DSSnapper SMedzhitov R. Anti-Inflammatory Effect of IL-10 Mediated by Metabolic Reprogramming of Macrophages. Science (2017) 356:513-9. doi: 10.1126/science.aal3535

7. Kabat AMPearce EJ. Inflammation by Way of Macrophage Metabolism. Science (2017) 356:488-9. doi: 10.1126/science.aan2691

8. Gellersen BBrosens JJ. Cyclic Decidualization of the Human Endometrium in Reproductive Health and Failure. Endocrine Rev (2014) 35:851-905. doi: 10.1210/er.2014-1045

9. Schumacher ASharkey DJRobertson SAZenclussen AC. Immune Cells at the Fetomaternal Interface: How the Microenvironment Modulates Immune the dominant role in establishing the maternal-fetal immune interface during gestation establishment? How does the maternal-fetal immune system initiate immune tolerance to the exemption of the embryo from invasive behavior? What methods can be used to detect the temporal and spatial tipping points at which immune tolerance occurs to determine where and when to take the appropriate clinical decisions to prevent the risk? Therefore, the development of new tools and experimental models is imperative. We can be confident that researchers will make considerable progress in understanding reproductive, immune metabolism shortly.

\section{AUTHOR CONTRIBUTIONS}

YW, TY, and LD conceived the original idea and the structure of the manuscript. YW, JD, and JL drafted the first version of the manuscript. YW and SC developed the figures. SL and $\mathrm{LH}$ critically provided critical feedback and helped shape the manuscript. LD, TY, and YZ supervised and revised the manuscript. All authors contributed to the article and approved the submitted version.

\section{FUNDING}

This work was supported by the following grants: the National Key Research and Development Program of China (No. 2018YFC1004601, 2018YFC1003900/04), and the National Natural Science Foundation of China (No. 81801540), and Shenzhen Natural Science Foundation (JCYJ20190813161801676).
Cells To Foster Fetal Development. JI (2018) 201:325-34. doi: 10.4049/ jimmunol.1800058

10. Thiele KDiao LArck PC. Immunometabolism, Pregnancy, and Nutrition. Semin Immunopathol (2018) 40:157-74. doi: 10.1007/s00281-017-0660-y

11. Murphy MPO’Neill LAJ. Krebs Cycle Reimagined: The Emerging Roles of Succinate and Itaconate as Signal Transducers. Cell (2018) 174:780-4. doi: 10.1016/j.cell.2018.07.030

12. Fan JKrautkramer KAFeldman JLDenu JM. Metabolic Regulation of Histone Post-Translational Modifications. ACS Chem Biol (2015) 10:95-108. doi: $10.1021 / \mathrm{cb} 500846 \mathrm{u}$

13. Voss KLarsen SESnow AL. Metabolic Reprogramming and Apoptosis Sensitivity: Defining the Contours of a T Cell Response. Cancer Lett (2017) 408:190-6. doi: 10.1016/j.canlet.2017.08.033

14. Rathmell JC. Metabolism and Autophagy in the Immune System: Immunometabolism Comes of Age. Immunol Rev (2012) 249:5-13. doi: 10.1111/j.1600-065X.2012.01158.x

15. Castello AHentze MWPreiss T. Metabolic Enzymes Enjoying New Partnerships as RNA-Binding Proteins. Trends Endocrinol Metab (2015) 26:746-57. doi: 10.1016/j.tem.2015.09.012

16. Lv YTariq MGuo XKanwal SEsteban MA. Intricacies in the Cross Talk Between Metabolic Enzymes, RNA, and Protein Translation. J Mol Cell Biol (2019) 11:813-5. doi: 10.1093/jmcb/mjz089

17. Papathanassiu AEKo J-HImprialou MBagnati MSrivastava PKVu HA, et al. BCAT1 Controls Metabolic Reprogramming in Activated Human Macrophages and is Associated With Inflammatory Diseases. Nat Commun (2017) 8:16040. doi: 10.1038/ncomms 16040 
18. Williams NCO'neill LAJ. A Role for the Krebs Cycle Intermediate Citrate in Metabolic Reprogramming in Innate Immunity and Inflammation. Front Immunol (2018) 9:141. doi: 10.3389/fimmu.2018.00141

19. Ferrick DANeilson ABeeson C. Advances in Measuring Cellular Bioenergetics Using Extracellular Flux. Drug Discovery Today (2008) 13:268-74. doi: 10.1016/j.drudis.2007.12.008

20. Kaplon JZheng LMeissl KChaneton BSelivanov VAMackay G, et al. A Key Role for Mitochondrial Gatekeeper Pyruvate Dehydrogenase in OncogeneInduced Senescence. Nature (2013) 498:109-12. doi: 10.1038/nature12154

21. Pelgrom LRvan der Ham AJEverts B. Analysis of TLR-Induced Metabolic Changes in Dendritic Cells Using the Seahorse XF(e)96 Extracellular Flux Analyzer. Methods Mol Biol (2016) 1390:273-85. doi: 10.1007/978-1-49393335-8_17

22. van der Windt GJWEverts BChang C-HCurtis JDFreitas TCAmiel E, et al. Mitochondrial Respiratory Capacity Is a Critical Regulator of CD8+ T Cell Memory Development. Immunity (2012) 36:68-78. doi: 10.1016/ j.immuni.2011.12.007

23. Calton EKKeane KNRaizel RRowlands JSoares MJNewsholme P. Winter to Summer Change in Vitamin D Status Reduces Systemic Inflammation and Bioenergetic Activity of Human Peripheral Blood Mononuclear Cells. Redox Biol (2017) 12:814-20. doi: 10.1016/j.redox.2017.04.009

24. Liu P-SHo P-C. Determining Macrophage Polarization Upon Metabolic Perturbation. Methods Mol Biol (2019) 1862:173-86. doi: 10.1007/978-14939-8769-6_13

25. Van Dilla MATrujillo TTMullaney PFCoulter JR. Cell Microfluorometry: A Method for Rapid Fluorescence Measurement. Science (1969) 163:1213-4. doi: $10.1126 /$ science. 163.3872 .1213

26. Köhler GMilstein C. Continuous Cultures of Fused Cells Secreting Antibody of Predefined Specificity. Nature (1975) 256:495-7. doi: 10.1038/256495a0

27. Artyomov MNVan den Bossche J. Immunometabolism in the Single-Cell Era. Cell Metab (2020) 32:710-25. doi: 10.1016/j.cmet.2020.09.013

28. Xue MWei WSu YJohnson DHeath JR. Supramolecular Probes for Assessing Glutamine Uptake Enable Semi-Quantitative Metabolic Models in Single Cells. J Am Chem Soc (2016) 138:3085-93. doi: 10.1021/jacs.5b12187

29. Han GSpitzer MHBendall SCFantl WJNolan GP. Metal-Isotope-Tagged Monoclonal Antibodies for High-Dimensional Mass Cytometry. Nat Protoc (2018) 13:2121-48. doi: 10.1038/s41596-018-0016-7

30. Evers TMJHochane MTans SJHeeren RMASemrau SNemes $P$, et al. Deciphering Metabolic Heterogeneity by Single-Cell Analysis. Anal Chem (2019) 91:13314-23. doi: 10.1021/acs.analchem.9b02410

31. Maby PCorneau AGalon J. Phenotyping of Tumor Infiltrating Immune Cells Using Mass-Cytometry (CyTOF). Methods Enzymol (2020) 632:339-68. doi: 10.1016/bs.mie.2019.07.025

32. Jha PKumar DDikshit RBudukh ABegum RSati P, et al. Automated Versus Physician Assignment of Cause of Death for Verbal Autopsies: Randomized Trial of 9374 Deaths in 117 Villages in India. BMC Med (2019) 17:116. doi: 10.1186/s12916-019-1353-2

33. Tweeddale HNotley-McRobb LFerenci T. Effect of Slow Growth on Metabolism of Escherichia Coli, as Revealed by Global Metabolite Pool ("Metabolome") Analysis. J Bacteriol (1998) 180:5109-16. doi: 10.1128/ JB.180.19.5109-5116.1998

34. Patti GJYanes OSiuzdak G. Innovation: Metabolomics: The Apogee of the Omics Trilogy. Nat Rev Mol Cell Biol (2012) 13:263-9. doi: 10.1038/nrm3314

35. Abuawad AMbadugha CGhaemmaghami AMKim D-H. Metabolic Characterisation of THP-1 Macrophage Polarisation Using LC-MS-Based Metabolite Profiling. Metabolomics (2020) 16:33. doi: 10.1007/s11306-02001656-4

36. Liang LRasmussen M-LHPiening BShen XChen SRöst H, et al. Metabolic Dynamics and Prediction of Gestational Age and Time to Delivery in Pregnant Women. Cell (2020) 181:1680-1692.e15. doi: 10.1016/j.cell.2020.05.002

37. Gowda GANDjukovic D. Overview of Mass Spectrometry-Based Metabolomics: Opportunities and Challenges. In: Raftery D, editor. Mass Spectrometry in Metabolomics Methods in Molecular Biology. New York, NY: Springer New York. (2014). p. 3-12. doi: 10.1007/978-1-4939-1258-2_1

38. Amantonico AUrban PLFagerer SRBalabin RMZenobi R. Single-Cell MALDI-MS as an Analytical Tool for Studying Intrapopulation Metabolic Heterogeneity of Unicellular Organisms. Anal Chem (2010) 82:7394-400. doi: $10.1021 /$ ac1015326
39. Guillaume-Gentil ORey TKiefer PIbáñez AJSteinhoff RBrönnimann R, et al. Single-Cell Mass Spectrometry of Metabolites Extracted From Live Cells by Fluidic Force Microscopy. Anal Chem (2017) 89:5017-23. doi: 10.1021/ acs.analchem.7b00367

40. Fisher GLBruinen ALOgrinc Potočnik NHammond JSBryan SRLarson PE, et al. A New Method and Mass Spectrometer Design for TOF-SIMS Parallel Imaging Ms/MS. Anal Chem (2016) 88:6433-40. doi: 10.1021/acs.analchem.6b01022

41. González-Domínguez RSayago AFernández-Recamales Á. Direct Infusion Mass Spectrometry for Metabolomic Phenotyping of Diseases. Bioanalysis (2017) 9:131-48. doi: 10.4155/bio-2016-0202

42. Zhang WRamautar R. CE-MS for Metabolomics: Developments and Applications in the Period 2018-2020. Electrophoresis (2021) 42:381-401. doi: 10.1002/elps.202000203

43. Duncan KDFyrestam JLanekoff I. Advances in Mass Spectrometry Based Single-Cell Metabolomics. Analyst (2019) 144:782-93. doi: 10.1039/ c8an01581c

44. Zhang CWang YWang FWang ZLu YXu Y, et al. Quantitative Profiling of Glycerophospholipids During Mouse and Human Macrophage Differentiation Using Targeted Mass Spectrometry. Sci Rep (2017) 7:412. doi: 10.1038/s41598-017-00341-2

45. Voss KHong HSBader JESugiura ALyssiotis CARathmell JC. A Guide to Interrogating Immunometabolism. Nat Rev Immunol (2021). doi: 10.1038/ s41577-021-00529-8

46. Verbist KCGuy CSMilasta SLiedmann SKamiński MMWang R, et al. Metabolic Maintenance of Cell Asymmetry Following Division in Activated T Lymphocytes. Nature (2016) 532:389-93. doi: 10.1038/ nature 17442

47. Argüello RJCombes AJChar RGigan J-PBaaziz AIBousiquot E, et al. SCENITH: A Flow Cytometry-Based Method to Functionally Profile Energy Metabolism With Single-Cell Resolution. Cell Metab (2020) 32:1063-1075.e7. doi: 10.1016/j.cmet.2020.11.007

48. Horowitz AStrauss-Albee DMLeipold MKubo JNemat-Gorgani NDogan OC, et al. Genetic and Environmental Determinants of Human NK Cell Diversity Revealed by Mass Cytometry. Sci Transl Med (2013) 5:208ra145. doi: $10.1126 /$ scitranslmed.3006702

49. Tannahill GMCurtis AMAdamik JPalsson-McDermott EMMcGettrick AFGoel G, et al. Succinate is an Inflammatory Signal That Induces IL-1 $\beta$ Through HIF-1 $\alpha$. Nature (2013) 496:238-42. doi: 10.1038/nature11986

50. Hartmann FJMrdjen DMcCaffrey EGlass DRGreenwald NFBharadwaj A, et al. Single-Cell Metabolic Profiling of Human Cytotoxic T Cells. Nat Biotechnol (2021) 39:186-97. doi: 10.1038/s41587-020-0651-8

51. Bulmer JNWilliams PJLash GE. Immune Cells in the Placental Bed. Int J Dev Biol (2010) 54:281-94. doi: 10.1387/ijdb.082763jb

52. Moffett-King A. Natural Killer Cells and Pregnancy. Nat Rev Immunol (2002) 2:656-63. doi: $10.1038 /$ nri886

53. Cartwright JEJames-Allan LBuckley RJWallace AE. The Role of Decidual NK Cells in Pregnancies With Impaired Vascular Remodelling. J Reprod Immunol (2017) 119:81-4. doi: 10.1016/j.jri.2016.09.002

54. Tao YLi Y-HPiao H-LZhou W-JZhang DFu Q, et al. CD56brightCD25+ NK Cells are Preferentially Recruited to the Maternal/Fetal Interface in Early Human Pregnancy. Cell Mol Immunol (2015) 12:77-86. doi: 10.1038/ cmi.2014.26

55. Yang-Feng TLLi SBLeung WYCarcangiu MLSchwartz PE. Trisomy 12 and K-Ras-2 Amplification in Human Ovarian Tumors. Int J Cancer (1991) 48:678-81. doi: 10.1002/ijc.2910480508

56. Parham P. The Genetic and Evolutionary Balances in Human NK Cell Receptor Diversity. Semin Immunol (2008) 20:311-6. doi: 10.1016/ j.smim.2008.10.002

57. Chazara OXiong SMoffett A. Maternal KIR and Fetal HLA-C: A Fine Balance. J Leukoc Biol (2011) 90:703-16. doi: 10.1189/jlb.0511227

58. Slattery KWoods EZaiatz-Bittencourt VMarks SChew SConroy M, et al. Tgfß Drives NK Cell Metabolic Dysfunction in Human Metastatic Breast Cancer. J Immunother Cancer (2021) 9(2):e002044. doi: 10.1136/jitc-2020002044

59. Donnelly RPLoftus RMKeating SELiou KTBiron CAGardiner CM, et al. Mtorc1-Dependent Metabolic Reprogramming is a Prerequisite for NK Cell Effector Function. J Immunol (2014) 193:4477-84. doi: 10.4049/ jimmunol.1401558 
60. Keating SEZaiatz-Bittencourt VLoftus RMKeane CBrennan KFinlay DK, et al. Metabolic Reprogramming Supports IFN- $\gamma$ Production by CD56bright NK Cells. J Immunol (2016) 196:2552-60. doi: 10.4049/jimmunol.1501783

61. Zheng XQian YFu BJiao DJiang YChen P, et al. Mitochondrial Fragmentation Limits NK Cell-Based Tumor Immunosurveillance. Nat Immunol (2019) 20:1656-67. doi: 10.1038/s41590-019-0511-1

62. Assmann NO’Brien KLDonnelly RPDyck LZaiatz-Bittencourt VLoftus RM, et al. Srebp-Controlled Glucose Metabolism is Essential for NK Cell Functional Responses. Nat Immunol (2017) 18:1197-206. doi: 10.1038/ni.3838

63. Kidani YElsaesser HHock MBVergnes LWilliams KJArgus JP, et al. Sterol Regulatory Element-Binding Proteins are Essential for the Metabolic Programming of Effector T Cells and Adaptive Immunity. Nat Immunol (2013) 14:489-99. doi: 10.1038/ni.2570

64. Michelet XDyck LHogan ALoftus RMDuquette DWei K, et al. Metabolic Reprogramming of Natural Killer Cells in Obesity Limits Antitumor Responses. Nat Immunol (2018) 19:1330-40. doi: 10.1038/s41590-018-0251-7

65. Loftus RMAssmann NKedia-Mehta NO’Brien KLGarcia AGillespie C, et al. Amino Acid-Dependent Cmyc Expression is Essential for NK Cell Metabolic and Functional Responses in Mice. Nat Commun (2018) 9:2341. doi: 10.1038/s41467-018-04719-2

66. Williams PJSearle RFRobson SCInnes BABulmer JN. Decidual Leucocyte Populations in Early to Late Gestation Normal Human Pregnancy. J Reprod Immunol (2009) 82:24-31. doi: 10.1016/j.jri.2009.08.001

67. Murray PJ. Macrophage Polarization. Annu Rev Physiol (2017) 79:541-66. doi: 10.1146/annurev-physiol-022516-034339

68. Jaiswal MKMallers TMLarsen BKwak-Kim JChaouat GGilman-Sachs A, et al. V-ATPase Upregulation During Early Pregnancy: A Possible Link to Establishment of an Inflammatory Response During Preimplantation Period of Pregnancy. Reproduction (2012) 143:713-25. doi: 10.1530/REP-12-0036

69. Hamilton SOomomian YStephen GShynlova OTower CLGarrod A, et al. Macrophages Infiltrate the Human and Rat Decidua During Term and Preterm Labor: Evidence That Decidual Inflammation Precedes Labor. Biol Reprod (2012) 86:39. doi: 10.1095/biolreprod.111.095505

70. Zhang Y-HHe MWang YLiao A-H. Modulators of the Balance Between M1 and M2 Macrophages During Pregnancy. Front Immunol (2017) 8:120. doi: 10.3389/fimmu.2017.00120

71. Tsao F-YWu M-YChang Y-LWu C-THo H-N. M1 Macrophages Decrease in the Deciduae From Normal Pregnancies But Not From Spontaneous Abortions or Unexplained Recurrent Spontaneous Abortions. J Formos Med Assoc (2018) 117:204-11. doi: 10.1016/j.jfma.2017.03.011

72. Gonzalez JMFranzke C-WYang FRomero RGirardi G. Complement Activation Triggers Metalloproteinases Release Inducing Cervical Remodeling and Preterm Birth in Mice. Am J Pathol (2011) 179:838-49. doi: 10.1016/j.ajpath.2011.04.024

73. Vishnyakova PElchaninov AFatkhudinov TSukhikh G. Role of the Monocyte-Macrophage System in Normal Pregnancy and Preeclampsia. Int J Mol Sci (2019) 20. doi: 10.3390/ijms20153695

74. Brown MBvon Chamier MAllam ABReyes L. M1/M2 Macrophage Polarity in Normal and Complicated Pregnancy. Front Immunol (2014) 5:606. doi: 10.3389/fimmu.2014.00606

75. Rodríguez-Prados J-CTravés PGCuenca JRico DAragonés JMartín-Sanz P, et al. Substrate Fate in Activated Macrophages: A Comparison Between Innate, Classic, and Alternative Activation. J Immunol (2010) 185:605-14. doi: 10.4049/jimmunol.0901698

76. Van den Bossche JBaardman JOtto NAvan der Velden SNeele AEvan den Berg SM, et al. Mitochondrial Dysfunction Prevents Repolarization of Inflammatory Macrophages. Cell Rep (2016) 17:684-96. doi: 10.1016/ j.celrep.2016.09.008

77. Wenes MShang MDi Matteo MGoveia JMartín-Pérez RSerneels J, et al. Macrophage Metabolism Controls Tumor Blood Vessel Morphogenesis and Metastasis. Cell Metab (2016) 24:701-15. doi: 10.1016/j.cmet.2016.09.008

78. Montenegro-Burke JRSutton JARogers LMMilne GLMcLean JAAronoff DM. Lipid Profiling of Polarized Human Monocyte-Derived Macrophages. Prostaglandins Other Lipid Mediat (2016) 127:1-8. doi: 10.1016/ j.prostaglandins.2016.11.002

79. Chen HLiu YLi DSong JXia M. PGC-1 $\beta$ Suppresses Saturated Fatty AcidInduced Macrophage Inflammation by Inhibiting TAK1 Activation. IUBMB Life (2016) 68:145-55. doi: 10.1002/iub.1470
80. Van den Bossche JBaardman Jde Winther MPJ. Metabolic Characterization of Polarized M1 and M2 Bone Marrow-Derived Macrophages Using Real-Time Extracellular Flux Analysis. JoVE (2015) 105:53424. doi: 10.3791/53424

81. Zhou DHuang CLin ZZhan SKong LFang C, et al. Macrophage Polarization and Function With Emphasis on the Evolving Roles of Coordinated Regulation of Cellular Signaling Pathways. Cell Signal (2014) 26:192-7. doi: 10.1016/j.cellsig.2013.11.004

82. Van Dyken SJLocksley RM. Interleukin-4- and Interleukin-13-Mediated Alternatively Activated Macrophages: Roles in Homeostasis and Disease. Annu Rev Immunol (2013) 31:317-43. doi: 10.1146/annurev-immunol032712-095906

83. Jha AKHuang SC-CSergushichev ALampropoulou VIvanova YLoginicheva E, et al. Network Integration of Parallel Metabolic and Transcriptional Data Reveals Metabolic Modules That Regulate Macrophage Polarization. Immunity (2015) 42:419-30. doi: 10.1016/j.immuni.2015.02.005

84. Xiao WChen PDong JWang RLuo B. Dietary Glutamine Supplementation Partly Reverses Impaired Macrophage Function Resulting From Overload Training in Rats. Int J Sport Nutr Exerc Metab (2015) 25:179-87. doi: 10.1123/ijsnem.2014-0118

85. Zeng WLiu ZLiu XZhang SKhanniche AZheng Y, et al. Distinct Transcriptional and Alternative Splicing Signatures of Decidual CD4+ T Cells in Early Human Pregnancy. Front Immunol (2017) 8:682. doi: 10.3389/ fimmu.2017.00682

86. Nancy PErlebacher A. T Cell Behavior at the Maternal-Fetal Interface. Int $J$ Dev Biol (2014) 58:189-98. doi: 10.1387/ijdb.140054ae

87. Lissauer DKilby MDMoss P. Maternal Effector T Cells Within Decidua: The Adaptive Immune Response to Pregnancy? Placenta (2017) 60:140-4. doi: 10.1016/j.placenta.2017.09.003

88. Bromley SKMempel TRLuster AD. Orchestrating the Orchestrators: Chemokines in Control of T Cell Traffic. Nat Immunol (2008) 9:970-80. doi: 10.1038/ni.f.213

89. MacIver NJMichalek RDRathmell JC. Metabolic Regulation of T Lymphocytes. Annu Rev Immunol (2013) 31:259-83. doi: 10.1146/ annurev-immunol-032712-095956

90. Du M-RDong LZhou W-HYan F-TLi D-J. Cyclosporin a Improves Pregnancy Outcome by Promoting Functions of Trophoblasts and Inducing Maternal Tolerance to the Allogeneic Fetus in Abortion-Prone Matings in the Mouse. Biol Reprod (2007) 76:906-14. doi: 10.1095/ biolreprod.106.056648

91. Ghaebi MNouri MGhasemzadeh AFarzadi LJadidi-Niaragh FAhmadi M, et al. Immune Regulatory Network in Successful Pregnancy and Reproductive Failures. BioMed Pharmacother (2017) 88:61-73. doi: 10.1016/j.biopha.2017.01.016

92. Huang XCai YDing MZheng BSun HZhou J. Human Chorionic Gonadotropin Promotes Recruitment of Regulatory $\mathrm{T}$ Cells in Endometrium by Inducing Chemokine CCL2. J Reprod Immunol (2020) 137:102856. doi: $10.1016 /$ j.jri.2019.102856

93. Guerin LRMoldenhauer LMPrins JRBromfield JJHayball JDRobertson SA. Seminal Fluid Regulates Accumulation of FOXP3+ Regulatory T Cells in the Preimplantation Mouse Uterus Through Expanding the FOXP3+ Cell Pool and CCL19-Mediated Recruitment. Biol Reprod (2011) 85:397-408. doi: 10.1095/biolreprod.110.088591

94. Lin YXu LJin HZhong YDi JLin Q. CXCL12 Enhances Exogenous CD4 $+\mathrm{CD} 25+\mathrm{T}$ Cell Migration and Prevents Embryo Loss in non-Obese Diabetic Mice. Fertil Steril (2009) 91:2687-96. doi: 10.1016/j.fertnstert.2008.01.109

95. Kallikourdis MAndersen KGWelch KABetz AG. Alloantigen-Enhanced Accumulation of CCR5+ "Effector"Regulatory T Cells in the Gravid Uterus. Proc Natl Acad Sci U.S.A. (2007) 104:594-9. doi: 10.1073/pnas.0604268104

96. Teles ASchumacher AKühnle M-CLinzke NThuere CReichardt $P$, et al. Control of Uterine Microenvironment by Foxp3(+) Cells Facilitates Embryo Implantation. Front Immunol (2013) 4:158. doi: 10.3389/fimmu.2013.00158

97. Quinn KHLacoursiere DYCui LBui JParast MM. The Unique Pathophysiology of Early-Onset Severe Preeclampsia: Role of Decidual T Regulatory Cells. J Reprod Immunol (2011) 91:76-82. doi: 10.1016/ j.jri.2011.05.006

98. D’Addio FRiella LVMfarrej BGChabtini LAdams LTYeung M, et al. The Link Between the PDL1 Costimulatory Pathway and Th17 in Fetomaternal Tolerance. J Immunol (2011) 187:4530-41. doi: 10.4049/jimmunol.1002031 
99. Tsuda SNakashima AShima TSaito S. New Paradigm in the Role of Regulatory T Cells During Pregnancy. Front Immunol (2019) 10:573. doi: 10.3389/fimmu.2019.00573

100. Molvarec ACzegle ISzijártó JRigó J. Increased Circulating Interleukin-17 Levels in Preeclampsia. J Reprod Immunol (2015) 112:53-7. doi: 10.1016/ j.jri.2015.05.007

101. Liu LHuang XXu CChen CZhao WLi D, et al. Decidual Cd8+T Cells Exhibit Both Residency and Tolerance Signatures Modulated by Decidual Stromal Cells. J Transl Med (2020) 18:221. doi: 10.1186/s12967-020-02371-3

102. van der Zwan ABi KNorwitz ERCrespo ÂCClaas FHJStrominger JL, et al. Mixed Signature of Activation and Dysfunction Allows Human Decidual CD8+ T Cells to Provide Both Tolerance and Immunity. Proc Natl Acad Sci U.S.A. (2018) 115:385-90. doi: 10.1073/pnas.1713957115

103. Xu Y-YWang S-CLin Y-KLi D-JDu M-R. Tim-3 and PD-1 Regulate CD8+ T Cell Function to Maintain Early Pregnancy in Mice. J Reprod Dev (2017) 63:289-94. doi: 10.1262/jrd.2016-177

104. Wang SSun FLi MQian JChen CWang M, et al. The Appropriate Frequency and Function of Decidual Tim-3+CTLA-4+CD8+ T Cells are Important in Maintaining Normal Pregnancy. Cell Death Dis (2019) 10:407. doi: 10.1038/ s41419-019-1642-x

105. Warburg O. On Respiratory Impairment in Cancer Cells. Science (1956) 124:269-70

106. Pearce ELPoffenberger MCChang C-HJones RG. Fueling Immunity: Insights Into Metabolism and Lymphocyte Function. Science (2013) 342:1242454. doi: $10.1126 /$ science. 1242454

107. Carr ELKelman AWu GSGopaul RSenkevitch EAghvanyan A, et al. Glutamine Uptake and Metabolism Are Coordinately Regulated by ERK/ MAPK During T Lymphocyte Activation. JI (2010) 185:1037-44. doi: 10.4049/jimmunol.0903586

108. Macintyre ANGerriets VANichols AGMichalek RDRudolph MCDeoliveira D, et al. The Glucose Transporter Glut1 is Selectively Essential for CD4 T Cell Activation and Effector Function. Cell Metab (2014) 20:61-72. doi: 10.1016/j.cmet.2014.05.004

109. Patsoukis NBardhan KChatterjee PSari DLiu BBell LN, et al. PD-1 Alters TCell Metabolic Reprogramming by Inhibiting Glycolysis and Promoting Lipolysis and Fatty Acid Oxidation. Nat Commun (2015) 6:6692. doi: $10.1038 /$ ncomms 7692

110. Michalek RDGerriets VAJacobs SRMacintyre ANMacIver NJMason EF, et al. Cutting Edge: Distinct Glycolytic and Lipid Oxidative Metabolic Programs are Essential for Effector and Regulatory CD4+ T Cell Subsets. J Immunol (2011) 186:3299-303. doi: 10.4049/jimmunol.1003613

111. Shi LZWang RHuang GVogel PNeale GGreen DR, et al. Hifl $\alpha$-Dependent Glycolytic Pathway Orchestrates a Metabolic Checkpoint for the Differentiation of TH17 and Treg Cells. J Exp Med (2011) 208:1367-76. doi: $10.1084 /$ jem. 20110278

112. Dang EVBarbi JYang H-YJinasena DYu HZheng Y, et al. Control of TH17/ Treg Balance by Hypoxia-Inducible Factor 1. Cell (2011) 146:772-84. doi: $10.1016 /$ j.cell.2011.07.033

113. Wu CYosef NThalhamer TZhu CXiao SKishi Y, et al. Induction of Pathogenic TH17 Cells by Inducible Salt-Sensing Kinase SGK1. Nature (2013) 496:513-7. doi: 10.1038/nature11984

114. Kleinewietfeld MManzel ATitze JKvakan HYosef NLinker RA, et al. Sodium Chloride Drives Autoimmune Disease by the Induction of Pathogenic TH17 Cells. Nature (2013) 496:518-22. doi: 10.1038/nature11868

115. Smith PMHowitt MRPanikov NMichaud MGallini CABohlooly-Y M, et al. The Microbial Metabolites, Short-Chain Fatty Acids, Regulate Colonic Treg Cell Homeostasis. Science (2013) 341:569-73. doi: 10.1126/science.1241165

116. Yang J-QKalim KWLi YZhang SHinge AFilippi M-D, et al. RhoA Orchestrates Glycolysis for TH2 Cell Differentiation and Allergic Airway Inflammation. J Allergy Clin Immunol (2016) 137:231-245.e4. doi: 10.1016/ j.jaci.2015.05.004

117. Delgoffe GMPollizzi KNWaickman ATHeikamp EMeyers DJHorton MR, et al. The Kinase mTOR Regulates the Differentiation of Helper T Cells Through the Selective Activation of Signaling by Mtorc1 and Mtorc2. Nat Immunol (2011) 12:295-303. doi: 10.1038/ni.2005

118. Mahnke JSchumacher VAhrens SKäding NFeldhoff LMHuber M, et al. Interferon Regulatory Factor 4 Controls TH1 Cell Effector Function and Metabolism. Sci Rep (2016) 6:35521. doi: 10.1038/srep35521
119. Pearce ELWalsh MCCejas PJHarms GMShen HWang L-S, et al. Enhancing CD8 T-Cell Memory by Modulating Fatty Acid Metabolism. Nature (2009) 460:103-7. doi: 10.1038/nature08097

120. Shackelford DBShaw RJ. The LKB1-AMPK Pathway: Metabolism and Growth Control in Tumour Suppression. Nat Rev Cancer (2009) 9:563-75. doi: $10.1038 / \mathrm{nrc} 2676$

121. Rao RRLi QOdunsi KShrikant PA. The mTOR Kinase Determines Effector Versus Memory CD8 + T Cell Fate by Regulating the Expression of Transcription Factors T-Bet and Eomesodermin. Immunity (2010) 32:6778. doi: 10.1016/j.immuni.2009.10.010

122. Araki KTurner APShaffer VOGangappa SKeller SABachmann MF, et al. mTOR Regulates Memory CD8 T-Cell Differentiation. Nature (2009) 460:108-12. doi: 10.1038/nature08155

123. Zhang LTschumi BOLopez-Mejia ICOberle SGMeyer MSamson G, et al. Mammalian Target of Rapamycin Complex 2 Controls CD8 T Cell Memory Differentiation in a Foxo1-Dependent Manner. Cell Rep (2016) 14:1206-17. doi: 10.1016/j.celrep.2015.12.095

124. Pollizzi KNPatel CHSun I-HOh M-HWaickman ATWen J, et al. Mtorc1 and Mtorc2 Selectively Regulate CD8 ${ }^{+} \mathrm{T}$ Cell Differentiation. J Clin Invest (2015) 125:2090-108. doi: 10.1172/JCI77746

125. De Rosa VGalgani MPorcellini AColamatteo ASantopaolo MZuchegna C, et al. Glycolysis Controls the Induction of Human Regulatory T Cells by Modulating the Expression of FOXP3 Exon 2 Splicing Variants. Nat Immunol (2015) 16:1174-84. doi: 10.1038/ni.3269

126. Kishore MCheung KCPFu HBonacina FWang GCoe D, et al. Regulatory T Cell Migration Is Dependent on Glucokinase-Mediated Glycolysis. Immunity (2018) 48:831-2. doi: 10.1016/j.immuni.2018.03.034

127. Blagih JHennequart MZani F. Tissue Nutrient Environments and Their Effect on Regulatory T Cell Biology. Front Immunol (2021) 12:637960. doi: 10.3389/fimmu.2021.637960

128. Wei RLai NZhao LZhang ZZhu XGuo Q, et al. Dendritic Cells in Pregnancy and Pregnancy-Associated Diseases. BioMed Pharmacother (2021) 133:110921. doi: 10.1016/j.biopha.2020.110921

129. Eisenbarth SC. Dendritic Cell Subsets in T Cell Programming: Location Dictates Function. Nat Rev Immunol (2019) 19:89-103. doi: 10.1038/s41577018-0088-1

130. Du M-RGuo P-FPiao H-LWang S-CSun CJin L-P, et al. Embryonic Trophoblasts Induce Decidual Regulatory T Cell Differentiation and Maternal-Fetal Tolerance Through Thymic Stromal Lymphopoietin Instructing Dendritic Cells. II (2014) 192:1502-11. doi: 10.4049/jimmunol.1203425

131. Karsten CMBehrends JWagner AKFuchs FFigge JSchmudde I, et al. DC Within the Pregnant Mouse Uterus Influence Growth and Functional Properties of Uterine NK Cells: Innate Immunity. Eur J Immunol (2009) 39:2203-14. doi: 10.1002/eji.200838844

132. Tagliani EShi CNancy PTay C-SPamer EGErlebacher A. Coordinate Regulation of Tissue Macrophage and Dendritic Cell Population Dynamics by CSF-1. J Exp Med (2011) 208:1901-16. doi: 10.1084/ jem.20110866

133. Liu SWei HLi YHuang CLian RXu J, et al. Downregulation of ILT4 ${ }^{+}$ Dendritic Cells in Recurrent Miscarriage and Recurrent Implantation Failure. Am J Reprod Immunol (2018) 80:e12998. doi: 10.1111/aji.12998

134. Ehrentraut SSauss KNeumeister RLuley LOettel AFettke F, et al. Human Miscarriage Is Associated With Dysregulations in Peripheral Blood-Derived Myeloid Dendritic Cell Subsets. Front Immunol (2019) 10:2440. doi: 10.3389/ fimmu. 2019.02440

135. Lu HHu R. The Role of Immunity in the Pathogenesis and Development of Pre-Eclampsia. Scand J Immunol (2019) 90. doi: 10.1111/sji.12756

136. Ellis JEAnsari AAFett JDCarraway RDRandall HWMosunjac MI, et al. Inhibition of Progenitor Dendritic Cell Maturation by Plasma From Patients With Peripartum Cardiomyopathy: Role in Pregnancy-Associated Heart Disease. Clin Dev Immunol (2005) 12:265-73. doi: 10.1080/ 17402520500304352

137. Guilliams MGinhoux FJakubzick CNaik SHOnai NSchraml BU, et al. Dendritic Cells, Monocytes and Macrophages: A Unified Nomenclature Based on Ontogeny. Nat Rev Immunol (2014) 14:571-8. doi: 10.1038/ nri3712

138. Darmochwal-Kolarz DRolinski JTabarkiewicz JLeszczynska-Gorzelak BBuczkowski JWojas K, et al. Myeloid and Lymphoid Dendritic Cells in 
Normal Pregnancy and Pre-Eclampsia: Dendritic Cells in Normal Pregnancy and Pre-Eclampsia. Clin Exp Immunol (2003) 132:339-44. doi: 10.1046/j.1365-2249.2003.02136.x

139. Kämmerer UEggert AOKapp MMcLellan ADGeijtenbeek TBHDietl J, et al. Unique Appearance of Proliferating Antigen-Presenting Cells Expressing DC-SIGN (CD209) in the Decidua of Early Human Pregnancy. Am J Pathol (2003) 162:887-96. doi: 10.1016/S0002-9440(10)63884-9

140. Hsu PSantner-Nanan BDahlstrom JEFadia MChandra APeek M, et al. Altered Decidual DC-SIGN+ Antigen-Presenting Cells and Impaired Regulatory T-Cell Induction in Preeclampsia. Am J Pathol (2012) 181:2149-60. doi: 10.1016/j.ajpath.2012.08.032

141. Huang CZhang HChen XDiao LLian RZhang X, et al. Association of Peripheral Blood Dendritic Cells With Recurrent Pregnancy Loss: A Case-Controlled Study. Am J Reprod Immunol (2016) 76:326-32. doi: 10.1111/aji.12550

142. Brombacher ECEverts B. Shaping of Dendritic Cell Function by the Metabolic Micro-Environment. Front Endocrinol (2020) 11:555. doi: $10.3389 /$ fendo.2020.00555

143. Lawless SJKedia-Mehta NWalls JFMcGarrigle RConvery OSinclair LV, et al. Glucose Represses Dendritic Cell-Induced T Cell Responses. Nat Commun (2017) 8:15620. doi: 10.1038/ncomms15620

144. Everts BAmiel Evan der Windt GJWFreitas TCChott RYarasheski KE, et al. Commitment to Glycolysis Sustains Survival of NO-Producing Inflammatory Dendritic Cells. Blood (2012) 120:1422-31. doi: 10.1182/blood-2012-03-419747

145. Kakazu EKondo YKogure TNinomiya MKimura OUeno Y, et al. Plasma Amino Acids Imbalance in Cirrhotic Patients Disturbs the Tricarboxylic Acid Cycle of Dendritic Cell. Sci Rep (2013) 3:3459. doi: 10.1038/srep03459

146. Woidacki KJensen FZenclussen AC. Mast Cells as Novel Mediators of Reproductive Processes. Front Immunol (2013) 4:29. doi: 10.3389/ fimmu.2013.00029
147. Woidacki KPopovic MMetz MSchumacher ALinzke NTeles A, et al. Mast Cells Rescue Implantation Defects Caused by C-Kit Deficiency. Cell Death Dis (2013) 4:e462-2. doi: 10.1038/cddis.2012.214

148. Meyer NWoidacki KMaurer MZenclussen AC. Safeguarding of Fetal Growth by Mast Cells and Natural Killer Cells: Deficiency of One Is Counterbalanced by the Other. Front Immunol (2017) 8:711. doi: 10.3389/fimmu.2017.00711

Conflict of Interest: Authors SL, LH and LD were employed by company Shenzhen Jinxin Medical Technology Innovation Center, Co., Ltd.

The remaining authors declare that the research was conducted in the absence of any commercial or financial relationships that could be construed as a potential conflict of interest.

Publisher's Note: All claims expressed in this article are solely those of the authors and do not necessarily represent those of their affiliated organizations, or those of the publisher, the editors and the reviewers. Any product that may be evaluated in this article, or claim that may be made by its manufacturer, is not guaranteed or endorsed by the publisher.

Copyright $\odot 2021$ Wei, Ding, Li, Cai, Liu, Hong, Yin, Zhang and Diao. This is an open-access article distributed under the terms of the Creative Commons Attribution License (CC BY). The use, distribution or reproduction in other forums is permitted, provided the original author(s) and the copyright owner(s) are credited and that the original publication in this journal is cited, in accordance with accepted academic practice. No use, distribution or reproduction is permitted which does not comply with these terms. 